OPEN ACCESS

Edited by:

Mohamed Abu-Farha,

Dasman Diabetes Institute, Kuwait

Reviewed by:

Caroline Hasselmann,

CHU Tours, France

Aaron Hanukoglu,

Tel Aviv University, Israel

*Correspondence:

Patricia Olivier

patricia.olivier.hsj@ssss.gouv.qc.ca

Specialty section:

This article was submitted to Diabetes,

a section of the journal

Frontiers in Endocrinology

Received: 19 December 2016 Accepted: 31 March 2017

Published: 24 April 2017

Citation:

Manousaki D, Deladoëy J, Geoffroy L and Olivier $P$ (2017) Continuous Subcutaneous Insulin Infusion in Children: A Pilot Study

Validating a Protocol to Avoid

Hypoglycemia at Initiation.

Front. Endocrinol. 8:84.

doi: 10.3389/fendo.2017.00084

\section{Continuous Subcutaneous Insulin Infusion in Children: A Pilot Study Validating a Protocol to Avoid Hypoglycemia at Initiation}

\author{
Despoina Manousaki ${ }^{1,2}$, Johnny Deladoëy ${ }^{1,2}$, Louis Geoffroy ${ }^{1,2}$ and Patricia Olivier ${ }^{1,2 *}$ \\ ${ }^{1}$ Endocrinology Service and Diabetes Unit, CHU Sainte-Justine, Montreal, QC, Canada, ${ }^{2}$ Department of Pediatrics, \\ University of Montreal, Montreal, QC, Canada
}

Background: The occurrence of hypoglycemia and hyperglycemia during the first days after transition to continuous subcutaneous insulin infusion (CSII) in patients with type 1 diabetes has not been systematically studied in children. The aim of this prospective study was to demonstrate that the protocol applied in our diabetes clinic is safe at CSII initiation in children.

Methods: We assessed 22 pediatric patients with type 1 diabetes, using continuous glucose monitoring (CGM) before and after CSII initiation ( \pm 3 days).

Results: After CSII initiation, there was no difference in the rates of hypoglycemic events expressed as relative rates (RRs) per person-reading ( $R R=0.85, p=0.52,95 \% \mathrm{Cl}$ 0.52-1.39), as well as in the number of prolonged hypoglycemic events ( $>1 \mathrm{~h})$ per day $(\mathrm{RR}=1.12, p=0.56,95 \% \mathrm{Cl} 0.75-1.68)$. We observed only a trend toward prolonged episodes of hyperglycemia after pump initiation $(R R=1.52, p=0.06,95 \% \mathrm{Cl} 0.97-2.35)$.

Conclusion: Our study is the first to assess, through CGM and in a prospective way, the impact of a CSII initiation protocol on glycemic values. Our protocol provides a safe model to avoid hypoglycemia at CSII initiation in children.

Clinical Trial Registration: www.ClinicalTrials.gov, identifier NCT01840358.

Keywords: continuous subcutaneous insulin infusion, pediatrics, hypoglycemia, continuous glucose monitoring, type 1 diabetes

\section{INTRODUCTION}

Continuous subcutaneous insulin infusion (CSII) is a highly accurate and flexible tool for administration of insulin. However, good glycemic control is often difficult to achieve even with CSII. Although previous pediatric and adult studies provided evidence of an increased risk of hypoglycemia with CSII (1-5), recent studies in children have documented a decrease in this risk (6-9) with an increase in the risk of episodes of ketoacidosis (7).

An increasing number of patients with type 1 diabetes mellitus (T1DM) under insulin injections will switch to CSII treatment during childhood (10). When transitioning to CSII, the medical team programs manually the initial parameters, according to the total daily insulin (TDI) dose and the current metabolic control of the patient. Next, the medical team adjusts the CSII parameters to reach an optimal glycemic control. Our local protocol for calculating the initial parameters of CSII therapy is shown in Figure 1. 
The evening before CSII initiation:

- Give intermediate-acting insulin (NPH) as usual, OR

- Reduce long-acting insulin (glargine or detemir) by half

- The patient receives no insulin and is fasting in the morning of the CSII initiation

1. Determine the total daily insulin dose (TDD)

Assess glycemic control (HbA1c) of the last 2 weeks:

a) Reduce the TDD by $20 \%$ if the control is too tight (recurrent hypoglycemias) OR if HbA1c $<8 \%$

b) Reduce the TDD by $10 \%$ if $\mathrm{HbA} 1 \mathrm{c}$ is in the $8-9 \%$ range

c) Hold the current TDD if HbA1c is in the $9-10.5 \%$ range

d) Increase the TDD by $10-20 \%$ if the HbA1c is $>10.5 \%$

2. Divide the TDD in basal and bolus insulins

a) $40-50 \%$ of the TDD as basal insulin:

- $\quad$ is divided by 24 for hourly rate

- change this hourly basal rate if the following phenomena are noted:

i. Dawn phenomenon : increase by $0.1-0.3$ units / h or $10-20 \%$ between 4 and $8 \mathrm{AM}$

ii. Nocturnal hypoglycemia : reduce by $10 \%$ from midnight until 4 to $8 \mathrm{AM}$

b) $50-60 \%$ of the TDI as bolus insulin, which is distributed over the three meals

3. Calculate the insulin bolus as ratios (units of insulin per grams of carbohydrates)

- Use the rule of $500^{1}$ or ratios already used by the patient

4. Determine the specific adjustments for hyperglycemia

- Apply the sensitivity factor already used by the patient $O R$

- Calculate a new one based on the rule of $100^{2}$

- Avoid corrections of hyperglycemia unless BG levels $>15 \mathrm{mmol} / \mathrm{l}(270 \mathrm{mg} / \mathrm{dl})$

5. Adjust the basal insulin rates during the first day after CSII initiation

- For patients on long-acting insulins, reduce the basal rate of the first 24 hours by $30 \%$

- For patients on intermediate-acting insulin, reduce the basal rate of the first $12-24$ hours by $30 \%$

FIGURE 1 | CHU Sainte-Justine protocol for calculation of insulin dose at continuous subcutaneous insulin infusion (CSII) initiation. ${ }^{1}$ Rule of 500 [used to calculate the carbohydrate factor (grams of carbohydrate per unit of insulin)]: 500 divided by the TDI (11). ${ }^{2}$ Rule of 100 (used to calculate the sensitivity factor): sensitivity factor equal to 100 divided by the total daily insulin (TDI) dose (11).

To date, there is no consensus on the initial insulin doses recommended when starting CSII; this practice varies between centers and depends on the local experience (12). We identified several adult and pediatric protocols in the literature (12-21), with additional guidelines published by the American Diabetes Association, ${ }^{1}$ in textbooks $(22,23)$, by tertiary centers, ${ }^{2}$ and by pharmaceutical manufacturers. ${ }^{3}$ However, to our knowledge, none of these protocols has been validated for safety in children in a prospective study.

When comparing the above protocols, we observe large variability in the method of calculation. While most of them propose a reduction in the TDI dose while transitioning to CSII, the insulin dose of our protocol is relatively lower. Although, as mentioned above, glycemic control with long-term CSII use has been extensively studied in the literature (1-9), only one pediatric study has retrospectively assessed insulin needs at CSII

${ }^{1}$ http://www.diabetes.org/living-with-diabetes/treatment-and-care/medication/ insulin/getting-started.html

${ }^{2}$ http://www.nhslanarkshire.org.uk/Services/Diabetes/Diabetes\%20Clinical\%20 Guidelines/Documents/Insulin\%20Pump\%20Therapy\%20May\%2014.pdf

${ }^{3} \mathrm{http} / / /$ www.professional.medtronicdiabetes.com/resources-download-library initiation without reporting in detail rates of hypoglycemia and hyperglycemia (18).

In this context, we designed a prospective, single-center study. The main objective was to assess the difference in the frequency of hypoglycemia before and after initiation of CSII. We hypothesized that the frequency of hypoglycemia would be increased in the first 3 days after initiation of CSII compared to the 3 days prior to initiation. A secondary objective was to evaluate the overall glycemic control in the first days after CSII initiation, taking into consideration both hypoglycemia and hyperglycemia rates. For both outcomes, we aimed to explore the interaction of potential confounders [age, sex, body mass index (BMI), duration of T1DM, TDI, HbA1c, and type of insulin regimen before CSII].

\section{MATERIALS AND METHODS}

\section{Protocol}

The eligibility of patients starting on CSII was defined according to the following inclusion criteria: (1) patients diagnosed with T1DM and followed at our center; (2) insulin needs of $>0.5 \mathrm{U} /$ $\mathrm{kg}$ /day at the time of enrollment, to avoid the honeymoon period $(24,25)$; (3) most recent value of HbAlc between 7 and $10 \%$ 
(5th and 85th percentiles of our population), to exclude patients prone to repeated hypo- or hyperglycemia; (4) patients living at a distance of less than $50 \mathrm{~km}$ from our center (to enable visits at home by the research nurse); and (5) informed written consent prior to the entry in the study.

Before starting on CSII, all patients and their parents attended a series of outpatient training courses. During one of these courses, a week prior to CSII initiation, they received written information explaining the study. The eligible patients and their parents were then contacted by a research nurse, and those willing to participate were enrolled in the study only after a written informed consent was obtained by the patients and the parents of all non-adult patients.

As part of this project, we required the use a continuous glucose monitoring (CGM) device [iPro ${ }^{\circledR} 2$ CGM System (Medtronic MiniMed, Northridge, CA, USA)] for 6 days (3 days prior and 3 days after insulin pump initiation). This type of censor is "blinded" to the patient [no possibility of instantaneous reading of the blood glucose (BG) values]. Thus, the patient cannot adjust the insulin doses according to the CGM measures using this device. The CGM sensors were installed at the participant's home by a research nurse. Then, participants were asked to continue measuring their BG levels using their meter as usually recommended, or a minimum of four times a day prior to, and a minimum of eight times per day after the CSII initiation. No changes in diet and exercise habits were required prior to CSII start. Participants were asked to document the following information: injections of bolus insulin, meals, physical activity, and hypoglycemia (symptomatic or severe). As typically recommended at CSII initiation, we required to avoid snacks containing carbohydrates and moderate to intense exercise that could change the basal metabolism during the first 3 days on CSII. CGM data were considered valid if we obtained a total recording time of at least $24 \mathrm{~h}$ before and $24 \mathrm{~h}$ after pump initiation.

To be consistent, all changes in insulin doses were performed according to the following rules: (i) in cases of hypoglycemia, clinicians were instructed to wait for three consecutive episodes of hypoglycemia below $4 \mathrm{mmol} / \mathrm{L}(72 \mathrm{mg} / \mathrm{dL})$ before decreasing the basal rate by $20 \%$ until the next morning at $8: 00$ a.m.; (ii) during episodes of hyperglycemia, we recommended to measure ketonemia if glucose values were $>15 \mathrm{mmol} / \mathrm{L}(270 \mathrm{mg} / \mathrm{dL})$ and to contact the diabetes clinic if abnormal; and (iii) to facilitate CSII adjustments, correction doses were recommended only if BG values were $>15 \mathrm{mmol} / \mathrm{L}$ before meals, according to a prescribed scale.

\section{Variables Analyzed}

The following data were obtained from patients' medical records: sex, age, weight, BMI, duration of T1DM, TDI, HbAlc prior to CSII initiation, previous type of insulin regimen, comorbidities, medications, and number of hospitalizations for severe hypoglycemia or ketoacidosis. HbA1c was measured by high-pressure liquid chromatography (Somagen Diagnostics Inc., Edmonton, $\mathrm{AB}$, Canada).

Continuous glucose monitoring values were compared with values obtained by the glucometer to ensure the reliability of the data. CGM glucose levels were analyzed according to the following definition criteria: hypoglycemia was defined as CGM reading of $<4 \mathrm{mmol} / \mathrm{L}$ and hyperglycemia as CGM reading of $>15 \mathrm{mmol} / \mathrm{L}$. Prolonged episodes (of either hypoglycemia or hyperglycemia) were defined as episodes of abnormal values lasting $>1 \mathrm{~h}$, corresponding to $\geq 12$ consecutive abnormal CGM glucose readings (the iPro ${ }^{\circledR} 2$ CGM automatically records an average glucose value every $5 \mathrm{~min}$ ).

\section{Data Analysis}

The rate of occurrence of events was defined as the number of abnormal CGM glucose readings ( $<4$ or $>15 \mathrm{mmol} / \mathrm{L}$ ) divided by the total number of CGM readings available for the period (i.e., 3 days pre- and post-installation of the CSII); the result was then expressed as number of abnormal readings per person-reading. For each participant, we compared the above results before and after CSII initiation, and their ratio was expressed as relative rate (RR). The same type of analysis was followed to compare rates of prolonged episodes of hypoglycemia and hyperglycemia before and after CSII initiation. A detailed description of the statistical tests used in our comparisons appears in Section "Appendix."

\section{RESULTS}

\section{Recruitment}

Among 103 patients with T1DM starting on CSII at our clinic between April 2013 and June 2014, 69 subjects met the eligibility criteria (Figure 2). Among the 69 eligible patients, 40 subjects accepted to participate and 29 refused. The main reasons for these refusals were time investment and absence of direct benefit from participating. Among the 40 obtained CGM recordings, 22 were finally included in the analysis. Ten recordings were rejected due to limited number of readings: five were completely void due to various reasons (e.g., CGM device never captured BG values, low battery), and another five were incomplete due to a technical failure or accidental disconnection of the CGM device. Finally, eight subjects were considered as drop-outs: three participants

103 patients with T1D initiating CSII treatment at our center from April 2013 to June 2014

\begin{tabular}{|c|c|c|}
\hline 工 & & \\
\hline & \multicolumn{2}{|c|}{34 patients did not fulfill the inclusion criteria: } \\
\hline 69 eligible patients & \multicolumn{2}{|c|}{-20 too far from our center $(>50 \mathrm{~km})$} \\
\hline 35 boys- 34 girls & \multicolumn{2}{|c|}{$\begin{array}{l}-11 \text { with } \mathrm{HbA} 1 \mathrm{c} \text { outside the accepted range } \\
(7-10 \%)\end{array}$} \\
\hline & \multicolumn{2}{|c|}{-3 patients in honeymoon phase } \\
\hline \multicolumn{2}{|c|}{$\begin{array}{l}40 \text { patients accepted to } \\
\text { participate }\end{array}$} & 29 patients refused to participate \\
\hline \multicolumn{3}{|c|}{ 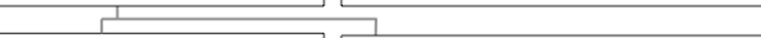 } \\
\hline \multicolumn{2}{|c|}{$\begin{array}{l}22 \text { recordings completed and } \\
\text { included in the study }\end{array}$} & $\begin{array}{l}18 \text { recordings excluded: } \\
-10 \text { void or incomplete recordings } \\
-8 \text { dropped-out after consent }\end{array}$ \\
\hline
\end{tabular}

FIGURE 2 | Breakdown of study subjects through inclusion and exclusion criteria. 
with an initially installed CGM were excluded as their physician decided that they were not ready for the CSII, and the remaining five subjects removed the CGM due to discomfort generated by the device (skin reaction, pain, and itchiness).

\section{Characteristics of Participants and Non-Participants}

Three groups were compared: participants who completed the study with appropriate CGM recordings $(n=22)$, participants who were excluded of the final analysis (insufficient CGM recordings or drop-outs, $n=18$ ), and patients who refused to participate $(n=29)$. The baseline characteristics of the three groups did not differ significantly. None of the 22 participants had a history of celiac or Addison's disease, or previous hospitalizations for episodes of severe hypoglycemia or ketoacidosis.

TABLE 1 | Comparison of baseline characteristics of the long-acting insulin group vs intermediate-acting insulin group.

\begin{tabular}{lccl}
\hline Baseline characteristics & $\begin{array}{c}\text { Long-acting } \\
\text { insulin group } \\
(\boldsymbol{n}=\mathbf{1 2})\end{array}$ & $\begin{array}{c}\text { Intermediate- } \\
\text { acting insulin } \\
\text { group }(\boldsymbol{n}=\mathbf{1 0})\end{array}$ & $\boldsymbol{p}$-Value \\
\hline Male $[n(\%)]$ & $6(50 \%)$ & $4(40 \%)$ & 0.29 \\
Age (years) & $13.36 \pm 3.72$ & $10.90 \pm 4.61$ & 0.19 \\
BMl (kg/m $\left.{ }^{2}\right)$ & $19.58 \pm 3.24$ & $18.64 \pm 2.91$ & 0.48 \\
Duration of diabetes (months) & $71.5(13-122)$ & $16.0(7-30)$ & $0.001^{\star}$ \\
TDI dose (U/kg/day) & $0.80(0.54-1.66)$ & $0.65(0.45-0.96)$ & 0.1 \\
HbA1c (\%) (mmol/mol) & $8.25 \pm 1.05$ & $7.83 \pm 0.90$ & 0.32 \\
& $66.7 \pm 11.5$ & $62.1 \pm 9.90$ & \\
\hline
\end{tabular}

${ }^{*} P$ values below 0.05 are denoted by an asterisk.

$B M I$, body mass index; TDI, total daily insulin.

Values are numbers and $\%$, means $\pm S D$, or median (range).

TABLE 2 | Frequency of abnormal readings and of prolonged episodes before and after continuous subcutaneous insulin infusion (CSII) initiation.

\begin{tabular}{llrrr}
\hline Variable & & $\begin{array}{c}\text { Before CSII } \\
\text { initiation }\end{array}$ & $\begin{array}{r}\text { After CSII } \\
\text { initiation }\end{array}$ & p-Value \\
\hline $\begin{array}{l}\text { Abnormal } \\
\text { readings per total }\end{array}$ & Hypoglycemia & $8.9(0-18.6)$ & $3.9(0-31.9)$ & 0.52 \\
$\begin{array}{l}\text { person-readings (\%) } \\
\text { Number of }\end{array}$ & Hypoglycemia & $1.0(0-3.8)$ & $1.0(0-3.6)$ & 0.57 \\
$\begin{array}{l}\text { prolonged episodes } \\
(>1 \text { h) per day }\end{array}$ & Hyperglycemia & $0.9(0-5.2)$ & $1.6(0-5.8)$ & 0.06 \\
\hline
\end{tabular}

Values are expressed as median (range).
Baseline characteristics of participants previously on longacting insulin $(n=12)$ and intermediate-acting insulin $(n=10)$ are shown in Table 1. Only duration of diabetes was different between the two groups, with the long-acting insulin group having a longer duration of diabetes (71.5 vs 16 months, $p=0.001$ ). This difference was not surprising, given that the majority of patients with newly diagnosed T1DM in our center start with an intermediate-acting insulin and switch to long-acting insulin later during their follow-up.

\section{Episodes of Prolonged Hypoglycemia and Hyperglycemia}

The average number of CGM glucose readings per patient was $693 \pm 220$ before CSII and $837 \pm 63$ after the CSII initiation. When we compared the values before and after CSII initiation, we found no difference in the rate of incidence of abnormal readings, in both hypoglycemic and hyperglycemic ranges, and in the number of prolonged episodes of hypoglycemia and hyperglycemia per day of recording (Table 2 ).

Also, there was no increase of the RR of prolonged hypoglycemic episodes after CSII initiation (Table 3). None of the participants presented severe hypoglycemia. Only one participant, formerly under long-acting insulin $\left(\right.$ Glargine $\left.^{\circledR}\right)$, required an insulin dose adjustment during the first $24 \mathrm{~h}$ after pump initiation, because of persistent hypoglycemia, which did not recur after the above adjustment. However, there was a trend to an increase of prolonged hyperglycemic episodes $[\mathrm{RR}=1.52(95 \%$ CI 0.99-2.35), $p=0.058$ ], with a median number of episodes per day of 0.9 before vs 1.6 after CSII initiation. None of the participants reported abnormally high blood ketones during the study duration. When we adjusted for potential confounders (sex, age, BMI, TDI, HbAlc, and type of insulin regimen before CSII), the increase of prolonged hyperglycemic episodes became statistically significant $[\mathrm{RR}=1.61(95 \% \mathrm{CI} 1.05-2.50), p=0.03]$ in the adjusted model.

\section{DISCUSSION}

The main objective of the present study was to evaluate the capacity of our CSII initiation protocol to prevent hypoglycemia at CSII initiation in children. To our knowledge, this is the first systematic prospective assessment of a protocol of insulin dose calculation at pump initiation. For this purpose, we used CGM to obtain BG readings before and after the start of CSII in the

TABLE 3 | RRs of abnormal readings and of prolonged episodes of hypoglycemia and hyperglycemia.

\begin{tabular}{|c|c|c|c|c|c|}
\hline \multirow[t]{2}{*}{ Variable } & & \multicolumn{2}{|c|}{$\mathbf{R R}^{\mathrm{b}}$ unadjusted } & \multicolumn{2}{|c|}{$\begin{array}{l}\mathrm{RR}^{\mathrm{b}} \text { adjusted for potential } \\
\text { confounders }\end{array}$} \\
\hline & & $\mathbf{R R}^{\mathrm{b}}(95 \% \mathrm{CI})$ & $p$-Value & $\mathbf{R R}^{\mathrm{b}}(95 \% \mathrm{Cl})$ & $p$-Value \\
\hline \multirow[t]{2}{*}{ Abnormal readings } & Hypoglycemia & $0.85(0.52-1.39)$ & 0.52 & $0.80(0.49-1.32)$ & 0.38 \\
\hline & Hyperglycemia & $1.26(0.81-1.97)$ & 0.31 & $1.32(0.87-2.01)$ & 0.19 \\
\hline \multirow[t]{2}{*}{ Prolonged episodes (>1 h) } & Hypoglycemia & $1.12(0.75-1.69)$ & 0.56 & $1.08(0.71-1.65)$ & 0.71 \\
\hline & Hyperglycemia & $1.52(0.99-2.35)$ & 0.06 & $1.61(1.05-2.50)$ & 0.03 \\
\hline
\end{tabular}

${ }^{a}$ Adjusted for sex, age, BMI, TDI dose, HbA1c, and type of insulin regimen before CSII.

${ }^{b} R R s$ after CSII/before CSII. Ratios >1.0 indicate higher risk compared to before CSII.

$R R$, relative rate; BMI, body mass index; TDI, total daily insulin; CSII, continuous subcutaneous insulin infusion. 
same patient. Therefore, each one of the 22 participants our study represents his/her own control. We considered this approach as a reliable method of comparing rates of hypoglycemia before and after CSII initiation.

Pediatric clinical studies implying additional burden (intervention such CGM device installation and time commitment) and few benefits have a notorious high refusal rate. Therefore, our present $42 \%$ refusal rate, while considered as a limitation of our study, is not surprising and is consistent with rates reported in previous pediatric studies (up to $51 \%$ ), although the latter studies required up to 6 months of real-time-CGM (RT-CGM) utilization $(26,27)$. We observed a high rate of unsuccessful recordings obtained with the use of a CGM, with only $57 \%$ of successful recordings. Similar problems, related to both standard CGM and RT-CGM use, have been described in the pediatric and adult population (28-30). Finally, our $20 \%$ drop-out rate (8 out of 40 patients) is equal to the ones reported in 2 pediatric studies requiring long-term CGM use $(28,31)$.

Our results indicated that there was no evidence of increase of hypoglycemia after CSII initiation. In contrast, an increase of hyperglycemia after CSII initiation was observed, which was more significant in the group receiving long-acting insulin. This finding was probably the consequence of the recommended $50 \%$ reduction of the dose of long-acting insulin at bedtime the day before CSII installation, whereas a decrease of the intermediateacting insulin dose was not recommended. Of note, we did not allow supplemental correction doses in the early days after pump installation for BG below $15 \mathrm{mmol} / \mathrm{L}$; the aim was to better understand the BG values and to better adjust the boluses and the basal rates. Furthermore, patients with newly installed CSII were asked to avoid moderate to intense exercise, which may explain the higher incidence of hyperglycemia in the days following the CSII initiation. Despite the increase in the episodes of prolonged hyperglycemia, the absence of reported high blood ketone levels during these episodes was reassuring in regards to the safety of our approach.

Our study has several limitations. First, given the lack of previous comparable studies, a proper power calculation to estimate the required number of patients to enroll was not possible. In this regard, our project should be considered as a pilot study. With the collection of a large number of glucose values per individual, our study had enough power to detect differences in the rates of prolonged hyperglycemia, and this suggests that the absence of difference in prolonged hypoglycemia is real and not due to missed true differences. Second, the CGM devices can fail to detect hypoglycemia. Many studies in adults suggest that the accuracy of the CGM data in the hypoglycemic range may be less optimal in T1DM patients $(32,33)$. According to Rebrin et al. (34), current sensors are generally less accurate in the first $24 \mathrm{~h}$ due to local tissue inflammation following trauma at the time of insertion, leading eventually to an underestimation of hypoglycemia in the first day after CGM initiation. To mitigate this risk, we decided to install the CGM device already 3 days before the CSII initiation. Moreover, we did not observe less hypoglycemia on the first day of the 22 recordings available for analysis. Another limitation of our study is the heterogeneity in the age of the participants. Indeed, differences in diurnal patterns in insulin needs have been reported in different age groups (13).
While the relatively small sample size of this study could not allow for stratification analysis, our findings suggest that our protocol is safe when applied in patients of different age groups. Finally, it is important to emphasize that our results, based on an observation period of 3 days after CSII initiation, are obviously not predictive of subsequent glycemic control and can only be used as a tool for initiation of CSII therapy.

\section{CONCLUSION}

Our pilot study is the first to assess, through CGM, and in a prospective way, the impact of a CSII installation protocol on glycemic values in the first days after CSII initiation. Despite the relatively increased frequency of hyperglycemia, our protocol does not lead to clinically relevant hypoglycemia and provides a safe model of calculating insulin doses for CSII in children. Further studies in larger numbers of patients, allowing for the evaluation of other protocols or the adaptation of the current one are needed, while new features in CSII devices, such as low glucose suspend, may further improve the safety at CSII initiation.

\section{ETHICS STATEMENT}

This project was approved by "the Research Ethics Committee of the CHU Sainte-Justine," and all research procedures were conducted in accordance with the recommendations of this Committee. All subjects gave written informed consent in accordance with the Declaration of Helsinki. This study was registered on www.clinicaltrials.org (NCT01840358). The protocol was approved by the "Commite d'Ethique du CHU Sainte-Justine."

\section{AUTHOR CONTRIBUTIONS}

DM contributed to the study concept and design, collected data, supervised the study, participated in data analysis and interpretation, and drafted, reviewed, and edited the manuscript. PO contributed to the study concept and design, collected data, supervised the study, participated in data analysis and interpretation, and reviewed and edited the manuscript. JD contributed to the study concept and design, participated in data analysis and interpretation, and reviewed and edited the manuscript. LG contributed to the study concept and design as well as reviewed and edited the manuscript.

\section{ACKNOWLEDGMENTS}

The authors thank the Unité de Recherche Clinique Appliquée (URCA) of the CHU Sainte-Justine for their contribution in the statistical analysis of our results, the research nurses (Mrs. Caroline Champagne, Mrs. Lise-Angela Ouellette, and Mrs. Maryse Thibeault) for technical assistance, and Dr. C. Deal for her critical review of the manuscript.

\section{FUNDING}

This work was supported by the Academic Funds of the Diabetes Unit of the CHU Sainte-Justine. JD is a junior 2 fellow of the Fonds de Recherche du Québec - Santé (FRQS). 


\section{REFERENCES}

1. Becker DJ, Ryan CM. Hypoglycemia: a complication of diabetes therapy in children. Trends Endocrinol Metab (2000) 11:198-202. doi:10.1016/S10432760(00)00259-9

2. Bulsara MK, Holman CD, Davis EA, Jones TW. The impact of a decade of changing treatment on rates of severe hypoglycemia in a population-based cohort of children with type 1 diabetes. Diabetes Care (2004) 27:2293-8. doi:10.2337/diacare.27.10.2293

3. Davis EA, Keating B, Byrne GC, Russell M, Jones TW. Impact of improved glycaemic control on rates of hypoglycaemia in insulin dependent diabetes mellitus. Arch Dis Child (1998) 78:111-5. doi:10.1136/adc.78.2.111

4. Donaghue KC, Fung AT, Hing S, Fairchild J, King J, Chan A, et al. The effect of prepubertal diabetes duration on diabetes. Microvascular complications in early and late adolescence. Diabetes Care (1997) 20:77-80. doi:10.2337/ diacare.20.1.77

5. The Diabetes Control and Complications Trial Research Group. Hypoglycemia in the diabetes control and complications trial. Diabetes (1997) 46:271-86. doi:10.2337/diab.46.2.271

6. Colino E, Martin-Frias M, Yelmo R, Alvarez MA, Roldan B, Barrio R. Impact of insulin pump therapy on long-term glycemic control in a pediatric Spanish cohort. Diabetes Res Clin Pract (2016) 113:69-76. doi:10.1016/j.diabres. 2016.01.012

7. Brorsson AL, Viklund G, Ortqvist E, Lindholm Olinder A. Does treatment with an insulin pump improve glycaemic control in children and adolescents with type 1 diabetes? A retrospective case-control study. Pediatr Diabetes (2015) 16:546-53. doi:10.1111/pedi.12209

8. Lau YN, Korula S, Chan AK, Heels K, Krass I, Ambler G. Analysis of insulin pump settings in children and adolescents with type 1 diabetes mellitus. Pediatr Diabetes (2016) 17:319-26. doi:10.1111/pedi.12285

9. Hanas R, Adolfsson P. Insulin pumps in pediatric routine care improve longterm metabolic control without increasing the risk of hypoglycemia. Pediatr Diabetes (2006) 7:25-31. doi:10.1111/j.1399-543X.2006.00145.x

10. Geoffroy L, Gonthier G. Le Diabète Chez L'enfant Et Ladolescent. Montreal, QC: CHU Sainte-Justine (2012).

11. Stoller WA. Individualizing insulin management. Three practical cases, rules for regimen adjustment. Postgrad Med (2002) 111:51-4, 59-60, 63-6. doi:10.3810/ pgm.2002.05.1197

12. Danne T, von Schutz W, Lange K, Nestoris C, Datz N, Kordonouri O. Current practice of insulin pump therapy in children and adolescents - the Hannover recipe. Pediatr Diabetes (2006) 7(Suppl 4):25-31. doi:10.1111/j.1399-543X. 2006.00166.x

13. Bachran R, Beyer P, Klinkert C, Heidtmann B, Rosenbauer J, Holl RW, et al. Basal rates and circadian profiles in continuous subcutaneous insulin infusion (CSII) differ for preschool children, prepubertal children, adolescents and young adults. Pediatr Diabetes (2012) 13:1-5. doi:10.1111/j.1399-5448. 2011.00777.x

14. Pankowska E, Blazik M, Dziechciarz P, Szypowska A, Szajewska H. Continuous subcutaneous insulin infusion vs. multiple daily injections in children with type 1 diabetes: a systematic review and meta-analysis of randomized control trials. Pediatr Diabetes (2009) 10:52-8. doi:10.1111/j.1399-5448.2008.00440.x

15. Ahern JA, Boland EA, Doane R, Ahern JJ, Rose P, Vincent M, et al. Insulin pump therapy in pediatrics: a therapeutic alternative to safely lower HbAlc levels across all age groups. Pediatr Diabetes (2002) 3:10-5. doi:10.1034/j.13995448.2002.30103.x

16. Doyle EA, Weinzimer SA, Steffen AT, Ahern JA, Vincent M, Tamborlane WV. A randomized, prospective trial comparing the efficacy of continuous subcutaneous insulin infusion with multiple daily injections using insulin glargine. Diabetes Care (2004) 27:1554-8. doi:10.2337/diacare.27.7.1554

17. Litton J, Rice A, Friedman N, Oden J, Lee MM, Freemark M. Insulin pump therapy in toddlers and preschool children with type 1 diabetes mellitus. J Pediatr (2002) 141:490-5. doi:10.1067/mpd.2002.127500

18. Nicolajsen T, Samuelsson A, Hanas R. Insulin doses before and one year after pump start: children have a reversed dawn phenomenon. J Diabetes Sci Technol (2012) 6:589-94. doi:10.1177/193229681200600314
19. Alemzadeh R, Hoffmann RG, Dasgupta M, Parton E. Development of optimal kids insulin dosing system formulas for young children with type 1 diabetes mellitus. Diabetes Technol Ther (2012) 14:418-22. doi:10.1089/dia.2011.0184

20. Klinkert C, Bachran R, Heidtmann B, Grabert M, Holl RW, Initiative DPV. Age-specific characteristics of the basal insulin-rate for pediatric patients on CSII. Exp Clin Endocrinol Diabetes (2008) 116:118-22. doi:10.1055/ s-2007-990296

21. Wizemann E, Renner R, Hepp K. Prospective evaluation of a standardized basal rate distribution for CSII in type 1 diabetes over 6 months. Diabetologie Stoffwechsel (2001) 10:57.

22. Bolderman K. Putting Your Patients on the Pump. Alexandria, VA: American Diabetes Association (2002).

23. Walsh J, Roberts R. Pumping Insulin. San Diego, CA: Torrey Pines Press (2000).

24. Aly $\mathrm{H}$, Gottlieb P. The honeymoon phase: intersection of metabolism and immunology. Curr Opin Endocrinol Diabetes Obes (2009) 16:286-92. doi:10.1097/MED.0b013e32832e0693

25. Abdul-Rasoul M, Habib H, Al-Khouly M. 'The honeymoon phase' in children with type 1 diabetes mellitus: frequency, duration, and influential factors. Pediatr Diabetes (2006) 7:101-7. doi:10.1111/j.1399-543X.2006.00155.x

26. Olivier P, Lawson ML, Huot C, Richardson C, Nakhla M, Romain J. Lessons learned from a pilot RCT of simultaneous versus delayed initiation of continuous glucose monitoring in children and adolescents with type 1 diabetes starting insulin pump therapy. J Diabetes Sci Technol (2014) 8:523-8. doi:10.1177/ 1932296814524855

27. Tsalikian E, Fox L, Weinzimer S, Buckingham B, White NH, Beck R, et al. Feasibility of prolonged continuous glucose monitoring in toddlers with type 1 diabetes. Pediatr Diabetes (2012) 13:301-7. doi:10.1111/j.1399-5448. 2011.00837.x

28. Messer L, Ruedy K, Xing D, Coffey J, Englert K, Caswell K, et al. Educating families on real time continuous glucose monitoring: the DirecNet navigator pilot study experience. Diabetes Educ (2009) 35:124-35. doi:10.1177/ 0145721708325157

29. Englert K, Ruedy K, Coffey J, Caswell K, Steffen A, Levandoski L, et al. Skin and adhesive issues with continuous glucose monitors: a sticky situation. J Diabetes Sci Technol (2014) 8:745-51. doi:10.1177/1932296814529893

30. Hoss U, Budiman ES, Liu H, Christiansen MP. Continuous glucose monitoring in the subcutaneous tissue over a 14-day sensor wear period. J Diabetes Sci Technol (2013) 7:1210-9. doi:10.1177/193229681300700511

31. Kordonouri O, Pankowska E, Rami B, Kapellen T, Coutant R, Hartmann R, et al. Sensor-augmented pump therapy from the diagnosis of childhood type 1 diabetes: results of the Paediatric Onset Study (ONSET) after 12 months of treatment. Diabetologia (2010) 53:2487-95. doi:10.1007/s00125-010-1878-6

32. Bay C, Kristensen PL, Pedersen-Bjergaard U, Tarnow L, Thorsteinsson B. Nocturnal continuous glucose monitoring: accuracy and reliability of hypoglycemia detection in patients with type 1 diabetes at high risk of severe hypoglycemia. Diabetes Technol Ther (2013) 15:371-7. doi:10.1089/ dia.2013.0004

33. Zijlstra E, Heise T, Nosek L, Heinemann L, Heckermann S. Continuous glucose monitoring: quality of hypoglycaemia detection. Diabetes Obes Metab (2013) 15:130-5. doi:10.1111/dom.12001

34. Rebrin K, Sheppard NF Jr, Steil GM. Use of subcutaneous interstitial fluid glucose to estimate blood glucose: revisiting delay and sensor offset. J Diabetes Sci Technol (2010) 4:1087-98. doi:10.1177/193229681000400507

Conflict of Interest Statement: The authors declare that the research was conducted in the absence of any commercial or financial relationships that could be construed as a potential conflict of interest.

Copyright $\odot 2017$ Manousaki, Deladoëy, Geoffroy and Olivier. This is an open-access article distributed under the terms of the Creative Commons Attribution License (CC $B Y)$. The use, distribution or reproduction in other forums is permitted, provided the original author(s) or licensor are credited and that the original publication in this journal is cited, in accordance with accepted academic practice. No use, distribution or reproduction is permitted which does not comply with these terms. 


\section{APPENDIX}

\section{Statistical Methods}

The rate of occurrence of hyperglycemic and hypoglycemic events was defined as the number of abnormal continuous glucose monitoring (CGM) glucose readings divided by the total number of CGM readings available for the period; the result was then expressed as number of abnormal readings per person-reading. The comparison of these results was expressed as a relative rate (RR), where the numerator is the number of abnormal readings per total number of person-readings after continuous subcutaneous insulin infusion (CSII) initiation, and the denominator is the number of abnormal readings per total number of person-readings before CSII initiation. The prolonged episodes of hypoglycemia and hyperglycemia $(>1 \mathrm{~h})$ are expressed as number of episodes per day of recording.
The RR for the prolonged hypoglycemic and hyperglycemic episodes represents the ratio of their incidence post-CSII to the incidence pre-CSII, respectively. As it was a paired test (the participant was his/her own control), all these variables were analyzed by a Poisson GEE with a "cluster" effect for the subject. Parameters that represented potential confounding variables (sex, age, body mass index, total daily insulin, HbAlc, and type of insulin regimen before CSII) were then added in the Poisson GEE model and, when the model indicated a possible interaction (defined as a level of significance corresponding to a $p<0.1$ ), a subgroup analysis was performed. Finally, for other analyses, we used Student's $t$-test for the normally distributed values, chi-squared to compare proportions, and ANOVA and Kruskal-Wallis for multiple comparisons. The analyses were performed using the SPSS 21 , and $p$-values $<0.05$ were considered significant. 